BMJ Open

Diabetes

Research

\& Care

\title{
Association between brachial-ankle pulse wave velocity and risk of type 2 diabetes mellitus: results from a cohort study
}

\author{
Yan-mei Lou, ${ }^{1}$ Min-qi Liao, ${ }^{2}$ Chang-yi Wang, ${ }^{3}$ Hong-en Chen, ${ }^{3}$ Xiao-lin Peng, ${ }^{3}$ \\ Dan Zhao, ${ }^{3}$ Xu-ping Gao, ${ }^{2}$ Shan Xu, ${ }^{3}$ Li Wang, ${ }^{3}$ Jian-ping Ma, ${ }^{3}$ Zhao Ping, ${ }^{1}$ \\ Fang-fang Zeng (D) ${ }^{2}$
}

\begin{abstract}
To cite: Lou Y, Liao M, Wang $\mathrm{C}$, et al. Association between brachial-ankle pulse wave velocity and risk of type 2 diabetes mellitus: results from a cohort study. BMJ Open Diab Res Care 2020;8:e001317. doi:10.1136/ bmjdrc-2020-001317
\end{abstract}

$\checkmark$ Additional material is published online only. To view please visit the journal online (http://dx.doi.org/10.1136/ bmjdrc-2020-001317).

Y-mL, M-qL and C-yW contributed equally.

Received 26 February 2020 Revised 10 June 2020 Accepted 12 June 2020

Check for updates

\section{(C) Author(s) (or their} employer(s)) 2020. Re-use permitted under CC BY-NC. No commercial re-use. See rights and permissions. Published by BMJ.

For numbered affiliations see end of article.

Correspondence to Dr Fang-fang Zeng; zengffjnu@126.com and Dr Zhao Ping;

jingyuting@sina.com

\section{ABSTRACT}

Introduction Brachial-ankle pulse wave velocity (baPWV), as a simple and easily measured marker of arterial stiffness, has not been prospectively explored for its role in type 2 diabetes mellitus (T2DM) risk among the general population. This study aimed to explore the association between baseline ba-PWV value and new-onset T2DM among Chinese adults.

Research design and methods Using data from Xiaotangshan Hospital, we conducted a prospective cohort study among those who underwent annual or biennial health check-up examinations and who had their baPWV measured from 2009 to 2016. We explored the risk of new-onset T2DM across ba-PWV tertiles using Cox proportional-hazards regression analysis.

Results 0 f 6122 adults (68.9\% male; mean age: 51.0 (SD 13.0) years) without T2DM and with ba-PWV measured at baseline, 599 participants developed T2DM during an average of 3.8 (SD 2.3) years of follow-up. After multivariable adjustment, ba-PWV was positively related to T2DM risk ( $p$ for trend=0.008). Compared with the lowest ba-PWV tertile, the HRs and their 95\% Cls were 1.57 (1.18 to 2.10 ) for the second and 1.66 (1.24 to 2.22) for the third tertile. The risk across ba-PWV tertiles increased steadily from $1000 \mathrm{~cm} / \mathrm{s}$ to $1400 \mathrm{~cm} / \mathrm{s}$ and then reached a plateau. Subgroup analyses indicated a significantly higher risk among those aged $<65$ years and current smokers ( $p$ for interactions: $<0.001$ and 0.006 ).

Conclusions Our findings suggest that ba-PWV might be a useful and independent predictor of new-onset T2DM with ba-PWV ranging between $1000 \mathrm{~cm} / \mathrm{s}$ and $1400 \mathrm{~cm} / \mathrm{s}$, especially among younger individuals and current smokers.

\section{INTRODUCTION}

The number of type 2 diabetes mellitus (T2DM) cases has quadrupled worldwide in the past three decades, and T2DM is the ninth major cause of death. ${ }^{1}$ With the rapid economic and demographic transitions in China, the number of T2DM cases in China has also increased substantially, with the prevalence increasing from $1 \%$ in the 1980 s to $11.0 \%$ (approximately 120.9 million)

\section{Significance of this study}

What is already known about this subject?

- Cardiovascular health has been proven to be associated with type 2 diabetes mellitus (T2DM).

What are the new findings?

- High brachial-ankle pulse wave velocity (ba-PWV) might increase T2DM risk.

- The J-shaped association between ba-PWV and T2DM might be aggravated by current smoking.

- The association between ba-PWV and T2DM might be more evident among younger individuals.

How might these results change the focus of research or clinical practice?

- Ba-PWV might be an independent predictor in the development of T2DM.

in $2017,,^{2}$ and China is considered a top epicenter for the global T2DM epidemic. This stresses the importance of identifying predictors to prevent the further increase in T2DM cases.

Pulse wave velocity (PWV) is a wellestablished surrogate of arterial stiffness (AS) ${ }^{4}$ and the carotid-femoral pulse wave velocity (cf-PWV), which measures the distance between the carotid and femoral arteries, has been associated with incident T2DM. However, the measurement of cf-PWV should be conducted by tonometry or Doppler, which requires specialized training, a long detecting duration and exposure of the sensitive part of the body. ${ }^{67}$ The methodological difficulties made it difficult to implement cf-PWV for large population screening. ${ }^{8}$ In the 2000s, measurement of the brachial-ankle pulse wave velocity (ba-PWV) was introduced into clinical practice in Japan. ${ }^{9}$ The ba-PWV measured four limbs by an oscillometric pressure sensor to reflect the stiffness of the 
aorta and peripheral arteries; thus, it could be directly measured and be a readily available method to be used in large samples. ${ }^{10}$ In addition, studies also reported that ba-PWV might be more representative in measuring the arterial load of the left ventricle than cf-PWV owing to its assessment of a greater extent of the arterial tree. ${ }^{11}$ Therefore, ba-PWV could be recognized as having an efficacy equal to that of cf-PWV. ${ }^{12}$ However, to our knowledge, no study has explored the predictive role of ba-PWV for T2DM risk among general population.

In view of the aforementioned factors, we aimed to assess whether ba-PWV is also an independent predictor of the development of T2DM in a large cohort of metabolically healthy men and women. If it is useful, ba-PWV may have public health implications due to its simplicity and ease of measurement.

\section{METHODS}

\section{Study population}

The details of the population have been described previously. ${ }^{13}$ An ongoing cohort study was initiated by Xiaotangshan Hospital (Beijing, China) on January 1, 2009. This study was based on men and women aged over 20 years who underwent an annual or biennial health examination at the clinics of Xiaotangshan Hospital. Among the included participants, the proportion of local government civil servants was $>90 \%$; the remaining approximately $10 \%$ of participants voluntarily paid for a health examination at the physical examination centere.

By December 31, 2016, there were 23431 to 32829 participants recorded in this database each year. Of the 52402 participants included in this cohort study, 45559 participants completed at least one follow-up. Participants were excluded from this analysis if they had T2DM or other major diseases, such as stroke, cancer, myocardial infarction or coronary heart disease, at baseline.

All procedures performed in studies involving human participants were conducted in accordance with the ethical standards of the institutional and/or national research committee and with the 1964 Helsinki declaration and its later amendments or comparable ethical standards. The requirement for informed consent was waived because only routine health screening data were used by us.

\section{Data collection}

\section{Anthropometric and laboratory measurements}

At each survey, the health examination comprised a standardized health questionnaire and a systematic physical examination completed by experienced nurses. The questionnaire was conducted through a face-to-face interview to collect information on sociodemographic characteristics (eg, age and sex), lifestyle habits (eg, smoking and alcohol drinking), medical history and disease history (eg, diabetes and cancers). Height and weight were measured and body mass index (BMI) was calculated. Blood pressure was measured after at least 5 min of rest.
After overnight fasting, blood samples were obtained from each subject. Fasting plasma glucose (FPG) was measured using the glucose dehydrogenase method (Merck, Darmstadt, Germany). Participants could also choose to undergo a 2-hour postprandial blood glucose test and have HbA1c measured by immunoassay on a DCA Vantage Analyzer.

\section{Measurement of ba-PWV}

Using the volume-plethysmographic apparatus (form PWV/ABI, Colin VP-1000, model BP-203RPE II), ba-PWV was measured with subjects in a supine position after they rested for at least $5 \mathrm{~min}$ to stabilize their heartbeat. The pulse curves from the brachial to ankle arteries of both sides were obtained with a pneumatic cuff connected to a plethysmographic sensor and an oscillometric pressure sensor to determine the volume pulse waveform and blood pressure placed on both the upper arms and ankles, respectively. The path length of the pulse wave was estimated based on the body height of the participant. ${ }^{9}$ The averaged ba-PWV values of the right and left sides were calculated and used for the main analyses. The coefficients of variation were $7.60 \%$ and $5.9 \%$ for left and right sides of ba-PWV, respectively.

\section{Ascertainment of incident of type 2 diabetes}

In each survey, participants were invited to provide their medical records (eg, diagnoses and hospital admissions) and participants were also interviewed about major health events, including diagnosed T2DM or ever/ current use of antidiabetic drugs. T2DM was defined as $\mathrm{FPG} \geq 7.0 \mathrm{mmol} / \mathrm{L}, \mathrm{HbAlc} \geq 48.0 \mathrm{mmol} / \mathrm{mol}(6.5 \%)$, a 2-hour postprandial plasma glucose $\geq 11.1 \mathrm{mmol} / \mathrm{L}$, a self-reported physician diagnosis of T2DM, or antidiabetic drug use at baseline and during the following-up period. ${ }^{514}$ All subjects without T2DM at baseline were followed from the date of the ba-PWV measurement until the first diagnosis of T2DM, loss to follow-up due to emigration or death, or end of follow-up, whichever came first.

\section{Statistical analysis}

Continuous variables that were less than $0.1 \%$ and greater than $99.9 \%$ were excluded as outliers. All analyses were conducted by using R V.3.5.1 (R Development Core Team, Vienna, Austria) software. Two-sided p value $<0.05$ was considered statistically significant.

Participants were divided into tertiles with respect to ba-PWV values: $\leq 1256,1257-1448$ and $>1448 \mathrm{~cm} / \mathrm{s}$. The person-years of follow-up for each subject was calculated from the date ba-PWV was first measured to the date of incident T2DM diagnosis or the last recorded date without the outcome event. Comparisons of general characteristics across ba-PWV tertiles were performed by general linear regression for continuous variables and by the Cochran-Armitage test for categorical variables.

Cox proportional-hazards regression models were used to analyze the association between ba-PWV and T2DM 
risk, with the lowest tertile as the reference group. We used three models to calculate HRs and their corresponding 95\% CIs. Model 1 included age and sex. Model 2 included the factors in model 1 plus marital status, smoking and drinking status, BMI, FPG, total cholesterol (TC) and high-density lipoprotein cholesterol (HDL-C). In model 3, we further explored the effect of adjustment for hypertension and heart rate because AS was considered a cause of hypertension ${ }^{7}$ and heart rate has been found to be associated with AS. ${ }^{15}$ To explore whether there was a non-linear association between ba-PWV and T2DM, natural cubic linear regression was adopted using the R smoothHR package. The optimal number of knots was based on minimizing Akaike's information criterion and 4 was the optimal number of knots for fixed effects in this study when estimating the smooth HR curve. ${ }^{16}$

In the fully adjusted model, subgroup analyses by sex (male vs female), age strata (20.0-44.9, 45.0-64.9 and $\geq 65.0$ years $)$, BMI $(18.0-23.9,24.0-27.9$ and $\geq 28.0 \mathrm{~kg} /$ $\left.\mathrm{m}^{2}\right)$, TC $(<5.06 \mathrm{vs} \geq 5.06 \mathrm{mmol} / \mathrm{L})$, and smoking and drinking status (never, past and current) were conducted. In addition, to assess the effect of inflammation on the association, high-sensitivity $\mathrm{C}$ reactive protein (hs-CRP) was added into the subgroup analyses with comparing the estimates for those with or without measurement of hs-CRP. We estimated the multiplicative interactions between ba-PWV and these factors by adding interaction terms and the presence of a significant interaction ( $p$ for interaction $<0.05$ ) means the dependence of the association between ba-PWV and T2DM risk on the presence of variation in these variables. ${ }^{17}$

\section{RESULTS}

Through December 31, 2016, a total of 52402 participants were initially examined (figure 1 ). With a lost-tofollow-up rate of $13.06 \%$, we excluded 6843 participants who had only been examined once and who were aged under 20 years. Moreover, 5487 participants with a disease history mentioned previously at baseline, 45 and 34 with outliers for glucose and ba-PWV, and 33792 with missing information for ba-PWV values were further excluded. Finally, 6122 eligible participants (4216 males and 1906 females) were included.

The mean age of the participants was 51.0 (SD 13.8) years and the median ba-PWV was 1338 (IQR 1210-1521) $\mathrm{cm} / \mathrm{s}$ at baseline. We identified 599 incident T2DM cases over an average of 3.8 (SD 2.3) person-years of follow-up. Table 1 shows the main characteristics of participants at baseline according to tertiles of ba-PWV.

Compared with participants in the lowest tertile, those in the highest tertile were more likely to be males, to be older and to have higher BMI, blood pressure, triglycerides, TC, low-density lipoprotein cholesterol (LDL-C), hs-CRP, 2-hour postprandial plasma glucose and $\mathrm{HbA1c}$ levels, whereas individuals with lower HDL-C levels were more common in the lower tertile.
As shown in table 2, elevated ba-PWV was significantly associated with an increased T2DM risk in models 1-3 (all $\mathrm{p}$ trend $<0.05$ ). This association was not substantially changed after further adjusting for additional covariates in model 3, and the HRs (95\% CI) for T2DM were 1.42 (1.09 to 1.86) for the second and 1.37 (1.02 to 1.85) for the third tertile compared with the first tertile ( $\mathrm{p}$ for trend $=0.044$ ). The results for the ba-PWV of the right or left sides were similar and consistent with the average ba-PWV (HRs for T3 vs T1: 1.41, $p$ for trend $=0.037$ for right side; 1.35 , $\mathrm{p}$ for trend $=0.045$ for left side).

The non-linear association between ba-PWV and T2DM risk is shown in figure 2. We observed a J-shaped association, and the risk increased dramatically with ba-PWV ranging between $1000 \mathrm{~cm} / \mathrm{s}$ and $1400 \mathrm{~cm} / \mathrm{s}$, and then the risk reached a plateau at ba-PWV $>1400 \mathrm{~cm} / \mathrm{s}$.

With adjustment for confounding variables, interaction terms were introduced into the Cox regression analysis. The maximally adjusted (model 3) interaction terms of age and smoking status exhibited significant effects on the relationship between ba-PWV and T2DM (see online supplementary table 1). The association between ba-PWV and T2DM was more apparent among those aged between 20.0 and 44.9 years and between 45.0 and 64.9 years than among those aged $\geq 65$ years (HRs for extreme tertiles: 3.15, 1.27, 1.07; $\mathrm{p}$ for interaction <0.01) (figure 3). We also observed a steadily increased correlation between age and ba-PWV (Spearman coefficient $r=0.62 ; \mathrm{p}<0.001$ ) (see online supplementary figure 1 ).

In addition, a more evident association was observed among the current smokers than among non-smokers and past smokers (HRs for extreme tertiles: 2.77, 1.15, 1.05; $\mathrm{p}$ for interaction=0.006) (figure 3; online supplementary table 1 and figure 2).

\section{DISCUSSION}

In this cohort study, we measured ba-PWV, as a measure of AS, in a large number of health examination participants $(\mathrm{n}=6122)$, and a J-shaped association was observed between ba-PWV and T2DM risk. The risk increased markedly in individuals with a ba-PWV between 1000 $\mathrm{cm} / \mathrm{s}$ and $1400 \mathrm{~cm} / \mathrm{s}$ and then reached a plateau. In addition, the risk was more evident among participants who were younger and current smoking. To our knowledge, this is the first report involving ba-PWV and T2DM risk in a general population-based cohort study.

AS has been suggested to be a predictor for the development of T2DM. ${ }^{18}$ Prenner and Chirinos ${ }^{18}$ summarized evidence related to AS and diabetes in 2015, and AS markers included pulse pressure, PWV, aortic characteristic impedance (Zc) and so on. ${ }^{18}$ Among these markers, PWV, which reflects the velocity of the pulse transmission time between two arterial points, is considered the non-invasive "gold standard" measurement of AS. The cf-PWV and the ba-PWV are the two most widely applied tools to measure AS. ${ }^{4}$ A recently published cohort study that included 2450 individuals who were followed for 
Screening sample: adult population who underwent annual or biennial health examinations at the clinic of the Xiaotangshan hospital from January 1, 2009 to December 31, $2016(\mathrm{n}=52402)$

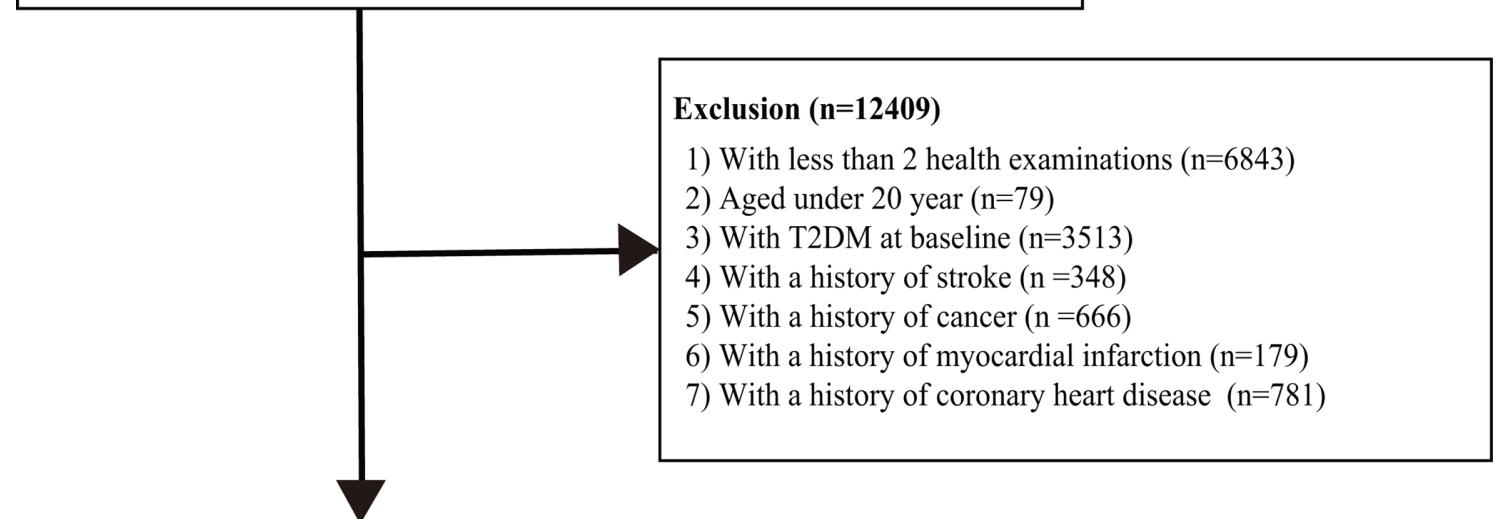

Eligible sample: adult aged 20 years old or older excluding missing values for T2DM diagnosis, T2DM at baseline and without history of stroke, cancer, myocardial infarction, and coronary heart disease $(n=39993)$

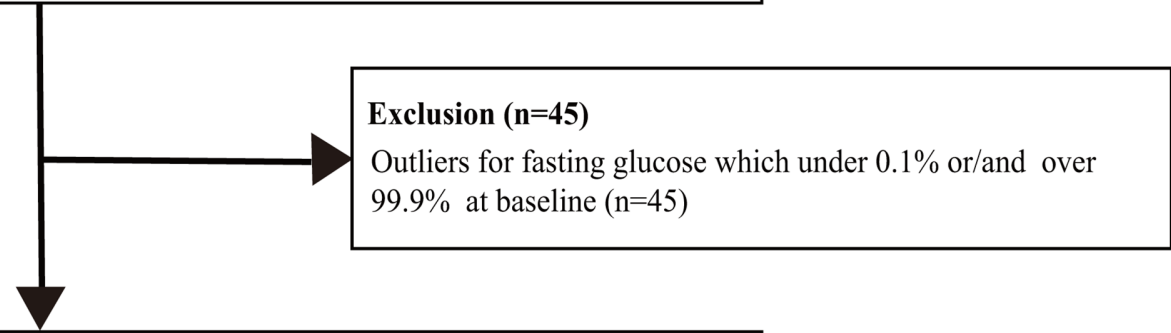

Eligible sample: adult aged 20 years or older excluding T2DM and other diseases at baseline, as well as excluding missing values and outliers of fast glucose $(\mathrm{n}=39948)$

Figure 1 Flow chart of population selection. ba-PWV, brachial-ankle pulse wave velocity; T2DM, type 2 diabetes mellitus.

approximately 4.4 years, with 68 incident diabetes cases, indicated that increased cf-PWV was associated with a $224 \%(95 \%$ CI $51 \%$ to $597 \%$ ) increased risk for diabetes when comparing the extreme tertiles. ${ }^{5}$ The cf-PWV measures the speed at which blood travels between the carotid and femoral arteries, assessing the real artery connecting these two points and evaluating large-artery stiffness. ${ }^{19}$ However, the measurement of cf-PWV, being conducted by tonometry or Doppler, requires specialized training, is time consuming and requires exposure of the sensitive part of the body, ${ }^{6}$ which restricts its routine clinical practice, as well as its use in large population screening. ${ }^{20}$ In contrast, the measurement of ba-PWV uses a separate cuff for each of the four limbs via an oscillometric pressure sensor to estimate the velocity through elastic and muscular arteries and then reflects the stiffness of the aorta and peripheral arteries in both sides of arms and legs, which could be measured via an automatic device and possesses a similar detection capability as cf-PWV. ${ }^{91012}$ Hence, the ba-PWV is a more favorable index to assess AS. 


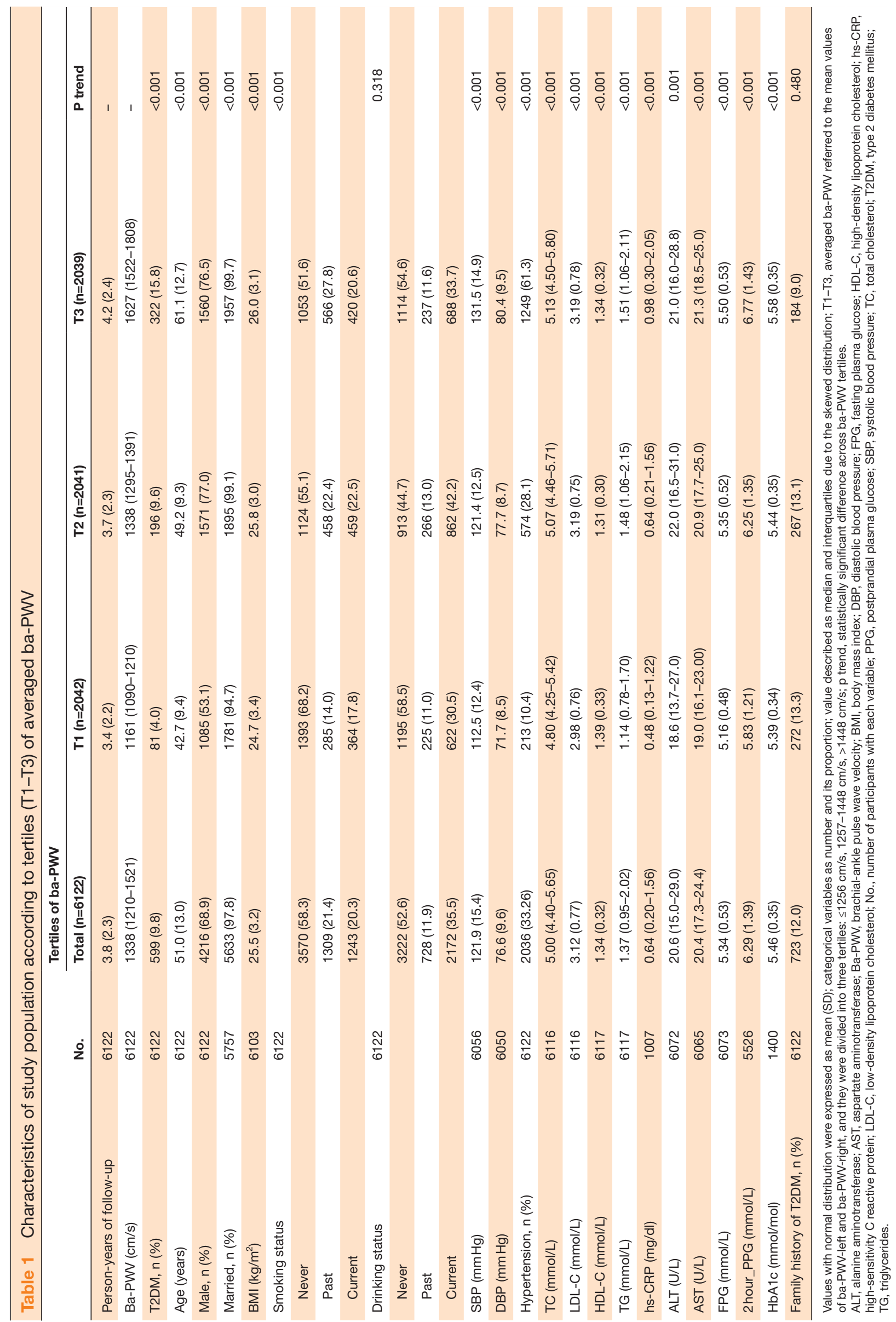




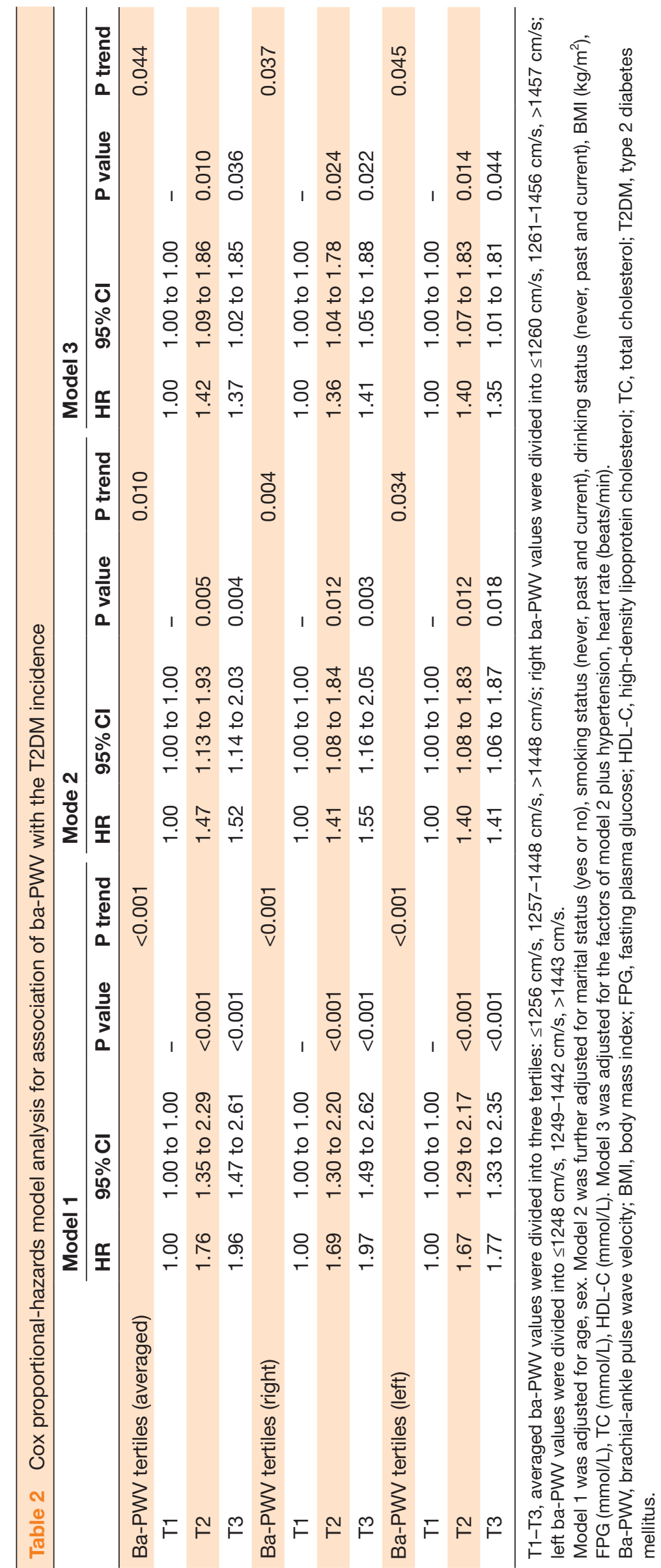

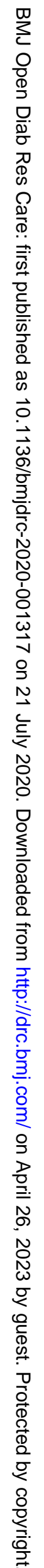




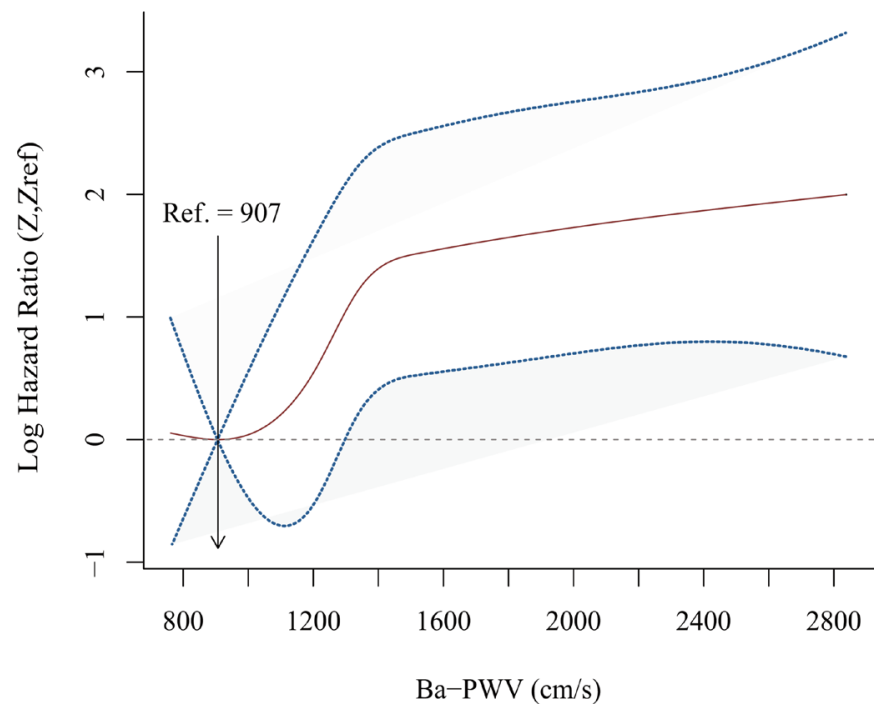

Figure 2 Shape of the association between baseline brachial-ankle pulse wave velocity (ba-PWV) and incidence of type 2 diabetes mellitus.

Few studies have investigated the effects of ba-PWV on diabetes-related markers. In a cross-sectional study, ${ }^{21}$ which was performed among 2188 Chinese middle-aged adults in 2004, ba-PWV was significantly higher in the diabetic group or impaired fasting glucose group than in the normoglycemic group. Similar findings were observed in another study with 4938 subjects recruited from the health examination center. ${ }^{22}$ In addition, a recent study published by Zhang $e t a l,{ }^{23}$ which followed a cohort of 2429 hypertensive participants for 4.5 years, with $287(11.8 \%)$ people developed into T2DM, showed that baseline ba-PWV was positively associated with the development of T2DM. Our finding further confirmed that elevated ba-PWV was significantly associated with increased T2DM risk among the general population.

Although the specific mechanism that links ba-PWV and T2DM remains unclear, the plausibility of a bidirectional relationship between AS and the risk of diabetes has been proposed by a previous study, ${ }^{24}$ and several potential mechanisms have been further proposed.
First, endothelial dysfunction refers to the inability of the endothelium to maintain vascular homeostasis and produce nitric oxide (NO), an endothelium-derived vasodilator that could augment arterial elasticity in the human brachial artery. ${ }^{25}$ Endothelial dysfunction is the shared pathophysiology of AS and T2DM, and it has been demonstrated to precede clinically evident T2DM. ${ }^{26}$ Second, microvascular rarefaction might directly reduce the uptake of glucose by the skeletal muscle, leading to reduced insulin sensitivity. ${ }^{27}$ Third, chronic inflammation might induce structural changes in the arterial wall by breaking down the elastin, affecting the proliferation of smooth muscle cells, and changing the composition of the extracellular matrix, ${ }^{28}$ thus facilitating the progression of AS. Similarly, inflammation may also induce metabolic changes and further lead to insulin resistance and T2DM. ${ }^{29}$ Thus, the chronic inflammation caused by AS might further accelerate the development of T2DM.

After stratifying by age, we found the association between ba-PWV and T2DM risk to be more substantial in younger patients than in older patients. This discrepancy is in accordance with our findings that when the ba-PWV was over $1400 \mathrm{~cm} / \mathrm{s}$, the risk remained almost stable as ba-PWV increased. According to our data, the ba-PWV increased with age, and the mean ba-PWV value was 1775 $\mathrm{cm} / \mathrm{s}$ for people aged $\geq 65$ years (data no shown), which is higher than $1400 \mathrm{~cm} / \mathrm{s}$, indicating that there were no longer increased risks associated with increased ba-PWV in this age group. The potential mechanism might be that endothelium-dependent NO dilatation might be damaged with age by producing increasing levels of reactive oxygen species, ${ }^{30}$ and it might result in higher ba-PWV and fasting blood glucose values among elderly individuals. Thus, the increasing trend of T2DM risk across ba-PWV tertiles might not be sensitive among the elderly population compared with among the younger population.

In addition, we also observed that the association between elevated ba-PWV and increased T2DM risk was more evident among current smokers than among

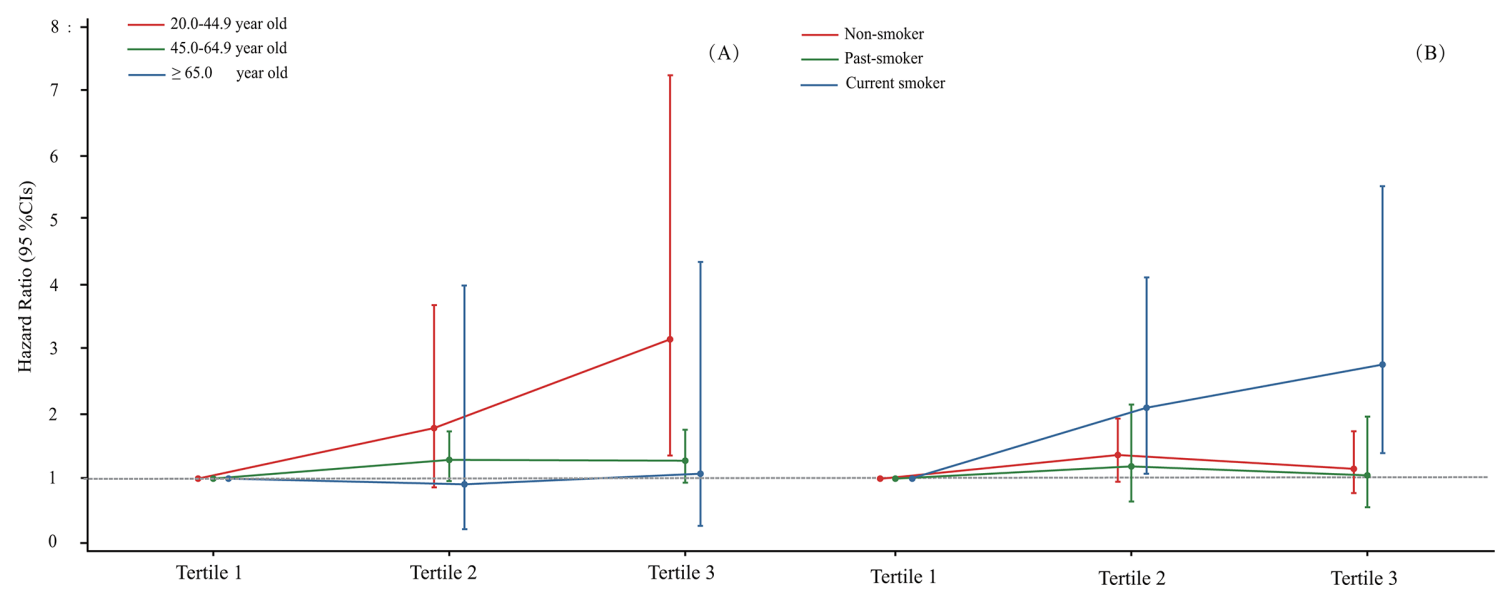

Figure 3 Type 2 diabetes mellitus risk according to baseline brachial-ankle pulse wave velocity levels across different age strata $(A)$ and smoking status $(B)$. 
non-smokers or past smokers. Multiple mechanisms underlie that tobacco smoking might increase the activation of Nox2-derived peptide, a marker of NADPH oxidase activation that could be responsible for endothelial dysfunction, inflammation, NO reduction and oxidative stress that lead to AS. ${ }^{31}$ Our finding agrees with previous evidence implying that smoking might exert a pro-inflammatory effect, and inhibit the exocrine and endocrine interactions of the pancreas, thus leading to damaged insulin sensitivity and pancreatic $\beta$-cell function. $^{3233}$

To explore whether the results were affected by inflammatory markers, hs-CRP was added to the subgroup analysis, but no obvious interaction was found. However, the evident gap in sample size between groups with and without hs-CRP data (1007 vs 5115) might limit the statistical power of hs-CRP data. Hence, further studies with adequate sample size with hs-CRP measurement are invited to fully investigate whether hs-CRP modified the association between ba-PWV and T2DM.

This cohort study included a relatively large sample that allowed enough power to estimate the strength of the associations. Nevertheless, several limitations need to be noted. First, our study was limited to health check-up participants in a hospital; thus, selection biases were inevitable. Second, error in ba-PWV measurement due to different operators or equipment could not be ignored. However, these measurement errors tend to underestimate the association. ${ }^{34}$ Third, although we adjusted for a wide range of factors, we could not rule out residual confounding. Finally, we only assessed the effects of baseline ba-PWV, so longitudinal changes need further confirmation.

In conclusion, we found that ba-PWV showed a J-shaped association with the development of T2DM in a Chinese adult population, and this association was prominent among younger people and current smokers. These results suggest that ba-PWV might be a useful early risk marker for predicting T2DM.

\section{Author affiliations}

${ }^{1}$ Department of Health Management, Beijing Xiao Tang Shan Hospital, Beijing, China

2Department of Epidemiology, Jinan University, Guangzhou, China

${ }^{3}$ Department of Non-communicable Disease Prevention and Control, Shenzhen

Nanshan Center for Chronic Disease Control, Shenzhen, China

Acknowledgements We would like to thank the staff and participants from the Beijing Xiao Tang Shan Hospital and the Shenzhen Nanshan Centre for Chronic Disease Control for their valuable contributions.

Contributors All listed authors meet the requirements for authorship. F-fZ and $Z P$ were responsible for study concept and design. $Y$ - $m L$ and $M-q L$ conceived the manuscript. C-yW, H-eC and X-IP performed the quality control. DZ, SX and J-pM contributed to the analysis plan and interpretations. X-pG and LW contributed to the formal data analysis. All the authors read and approved the final manuscript.

Funding This study was funded by the National Natural Science Foundation of China (grant no. 81602853) and Youth Top-notch Personal Project from Fangshan District Excellent Talent Cultivation Funds (grant no. 2016000000007B001).

Competing interests None declared.

Patient consent for publication Not required.

Ethics approval Institutional Review Board of Xiaotangshan Hospital (no. 202006).
Provenance and peer review Not commissioned; externally peer reviewed.

Data availability statement No data are available. None.

Open access This is an open access article distributed in accordance with the Creative Commons Attribution Non Commercial (CC BY-NC 4.0) license, which permits others to distribute, remix, adapt, build upon this work non-commercially, and license their derivative works on different terms, provided the original work is properly cited, appropriate credit is given, any changes made indicated, and the use is non-commercial. See: http://creativecommons.org/licenses/by-nc/4.0/.

\section{ORCID iD}

Fang-fang Zeng http://orcid.org/0000-0001-8650-0927

\section{REFERENCES}

1 Zheng Y, Ley SH, Hu FB. Global aetiology and epidemiology of type 2 diabetes mellitus and its complications. Nat Rev Endocrinol 2018;14:88-98.

2 Gu D, Reynolds K, Duan X, et al. Prevalence of diabetes and impaired fasting glucose in the Chinese adult population: international collaborative study of cardiovascular disease in Asia (InterASIA). Diabetologia 2003;46:1190-8.

3 International Diabetes Federation. IDF Diabetes Atlas. 8th edn. Brussels, Belgium: International Diabetes Federation, 2017. http:// www.diabetesatlas.org. (accessed 20 December 2019).

4 Lu Y, Zhu M, Bai B, et al. Comparison of carotid-femoral and brachial-ankle pulse-wave velocity in association with target organ damage in the community-dwelling elderly Chinese: the Northern Shanghai study. J Am Heart Assoc 2017;6:e004168.

5 Muhammad IF, Borné Y, Östling G, et al. Arterial stiffness and incidence of diabetes: a population-based cohort study. Diabetes Care 2017;40:1739-45.

6 Mancia G, Fagard R, Narkiewicz K, et al. 2013 ESH/ESC Guidelines for the management of arterial hypertension: the Task Force for the management of arterial hypertension of the European Society of Hypertension (ESH) and of the European Society of Cardiology (ESC). J Hypertens 2013;31:1281-357.

7 Townsend RR, Wilkinson IB, Schiffrin EL, et al. Recommendations for improving and standardizing vascular research on arterial stiffness: a scientific statement from the American Heart Association. Hypertension 2015;66:698-722.

8 Munakata M. Brachial-ankle pulse wave velocity: background, method, and clinical evidence. Pulse 2016;3:195-204.

9 Yamashina A, Tomiyama H, Takeda K, et al. Validity, reproducibility, and clinical significance of noninvasive brachial-ankle pulse wave velocity measurement. Hypertens Res 2002;25:359-64.

10 Tsuchikura S, Shoji T, Kimoto E, et al. Brachial-ankle pulse wave velocity as an index of central arterial stiffness. J Atheroscler Thromb 2010;17:658-65.

11 Yu W-C, Chuang S-Y, Lin Y-P, et al. Brachial-ankle vs carotid-femoral pulse wave velocity as a determinant of cardiovascular structure and function. J Hum Hypertens 2008;22:24-31.

12 Zhang Y, Agnoletti D, Xu Y, et al. Carotid-femoral pulse wave velocity in the elderly. J Hypertens 2014;32:1572-6.

$13 \mathrm{Hu}$ F, Lou Y, Shi J, et al. Baseline serum albumin and its dynamic change is associated with type 2 diabetes risk: a large cohort study in China. Diabetes Metab Res Rev 2020;36:e3296.

14 Xu M, Huang Y, Xie L, et al. Diabetes and risk of arterial stiffness: a Mendelian randomization analysis. Diabetes 2016;65:1731-40.

15 Lantelme $\mathrm{P}$, Mestre $\mathrm{C}$, Lievre $\mathrm{M}$, et al. Heart rate: an important confounder of pulse wave velocity assessment. Hypertension 2002;39:1083-7.

16 Meira-Machado L, Cadarso-Suárez C, Gude F, et al. smoothHR: an $\mathrm{R}$ package for pointwise nonparametric estimation of hazard ratio curves of continuous predictors. Comput Math Methods Med 2013;2013:1-11.

17 Al-Khadra AS, Salem DN, Rand WM, et al. Warfarin anticoagulation and survival: a cohort analysis from the studies of left ventricular dysfunction. J Am Coll Cardiol 1998;31:749-53.

18 Prenner SB, Chirinos JA. Arterial stiffness in diabetes mellitus. Atherosclerosis 2015;238:370-9.

19 Hashimoto J, Ito S. Central pulse pressure and aortic stiffness determine renal hemodynamics: pathophysiological implication for microalbuminuria in hypertension. Hypertension 2011;58:839-46.

20 Van Bortel LM, Laurent S, Boutouyrie P, et al. Expert consensus document on the measurement of aortic stiffness in daily practice using carotid-femoral pulse wave velocity. $J$ Hypertens 2012;30:445-8.

21 Ho C-T, Lin C-C, Hsu H-S, et al. Arterial stiffness is strongly associated with insulin resistance in Chinese-a population-based 
study (Taichung Community Health Study, TCHS). J Atheroscler Thromb 2011;18:122-30.

$22 \mathrm{Li} \mathrm{C-H,} \mathrm{Wu} \mathrm{J-S,} \mathrm{Yang} \mathrm{Y-C,} \mathrm{et} \mathrm{al.} \mathrm{Increased} \mathrm{arterial} \mathrm{stiffness} \mathrm{in}$ subjects with impaired glucose tolerance and newly diagnosed diabetes but not isolated impaired fasting glucose. J Clin Endocrinol Metab 2012;97:E658-62.

23 Zhang Y, He P, Li Y, et al. Positive association between baseline brachial-ankle pulse wave velocity and the risk of newonset diabetes in hypertensive patients. Cardiovasc Diabetol 2019;18:111-9.

24 Weber T. Arterial stiffness, wave reflections, and diabetes: a bidirectional relationship? Am J Hypertens 2010;23:1047-8.

25 Kinlay S, Creager MA, Fukumoto M, et al. Endothelium-derived nitric oxide regulates arterial elasticity in human arteries in vivo. Hypertension 2001;38:1049-53.

26 Giraldo-Grueso M, Echeverri D. From endothelial dysfunction to arterial stiffness in diabetes mellitus. Curr Diabetes Rev 2018. [Epub ahead of print: 17 Oct 2018].

27 Levy BI, Schiffrin EL, Mourad J-J, et al. Impaired tissue perfusion: a pathology common to hypertension, obesity, and diabetes mellitus. Circulation 2008;118:968-76.
28 Jain S, Khera R, Corrales-Medina VF, et al. Inflammation and arterial stiffness in humans. Atherosclerosis 2014;237:381-90.

29 Naidoo V, Naidoo M, Ghai M. Cell- and tissue-specific epigenetic changes associated with chronic inflammation in insulin resistance and type 2 diabetes mellitus. Scand J Immunol 2018;88:e12723.

30 Flavahan S, Chang F, Flavahan NA. Local renin-angiotensin system mediates endothelial dilator dysfunction in aging arteries. Am J Physiol Heart Circ Physiol 2016;311:H849-54.

31 Carnevale R, Sciarretta S, Violi F, et al. Acute impact of tobacco vs electronic cigarette smoking on oxidative stress and vascular function. Chest 2016;150:606-12.

32 Śliwińska-Mossoń M, Milnerowicz S, Milnerowicz H. Diabetes mellitus secondary to pancreatic diseases (type $3 c$ ): the effect of smoking on the exocrine-endocrine interactions of the pancreas. Diab Vasc Dis Res 2018;15:243-59.

33 Maddatu J, Anderson-Baucum E, Evans-Molina C. Smoking and the risk of type 2 diabetes. Transl Res 2017;184:101-7.

34 Spiegelman D. Approaches to uncertainty in exposure assessment in environmental epidemiology. Annu Rev Public Health 2010;31:149-63. 